\title{
Humidity Sensors Using Chemically Modified Polymer Thin Films
}

\author{
Yoshiro SAKAI* and Masanobu MATSUGUCHI
}

\author{
Department of Applied Chemistry, Faculty of Engineering, Ehime University (Matsuyama 790-8577, Japan)
}

Received July 5, 1999 ; Accepted July, 14, 1999

\begin{abstract}
Most of the humidity sensors practically used are based on the change in electrical properties of polymers or ceramics with humidity. They are either resistive-type or capacitive-type sensors. Various chemical modifications of polymeric materials have been utilized to improve the properties of sensors such as sensitivity, hysteresis, long-term stability, durability in unfavorable atmospheres such as dewdrops or vapors of volatile organic compounds(VOC), etc. This paper overviews the novel methods of chemical modification which have been proposed by the authors.
\end{abstract}

Key Words : Humidity Sensor, Polymer, Impedance, Capacitance

\section{Introduction}

Since the invention of an electrical humidity sensor by Dunmore based on $\mathrm{LiCl}$, various types of materials such as ceramics, metal oxide films, organic materials and polymers have been studied as materials for fabricating humidity sensors. Recently, polymeric materials have attracted more attention because of their long-term stability, reliability, ease of processing and the low cost of fabrication, etc. Most of the humidity sensors studied so far are based on either resistance or capacitance change of the materials caused by the sorption of moisture in them.

The humidity sensors fabricated using polymeric materials are classified into two categories, that is, the resistive-type and the capacitive-type. The former type is based on the variation in resistance (or impedance) with humidity, and the latter is based on the capacitance change. Resistive-type sensors have been fabricated with hydrophilic materials such as mixtures or complexes of inorganic salts with polymers or polymer electrolytes comprising cationic and anionic polymers. On the other hand, capacitive humidity sensors have been fabricated with hydrophobic polymers which sorb a very small amount of water vapor. In the early stage of development of practical humidity sensors, commercially available polymers were mainly used without any modification. In order to improve the sensors, we have proposed several methods of chemical modification of polymers to obtain more satisfactory materials for humidity sensors.

\section{Resistive-Type Humidity Sensors \\ 2. 1 Mixtures of polymer and inorganic salts}

We have found that polyethylene oxide (PEO) doped with various alkali salts such as $\mathrm{LiClO}_{4}$ shows a good impedance variation without any hysteresis in the range of $10^{7}$ to $10^{3} \Omega$ with humidification and desiccation and also has good sensitivity over the whole humidity range. ${ }^{1)}$ The salts form complexes with PEO. It was found that these complexes have low resistivity and low activation energy for electrical conduction. The free volume in PEO increases on sorbing water molecules, causing the hopping migration of cations. ${ }^{2)}$

\section{2 Humidity sensor using polymer electrolytes and their salts}

Various types of polymer electrolytes have been synthesized in recent years. They have hydrophilic groups such as $-\mathrm{COOH},-\mathrm{SO}_{3} \mathrm{H}$ or $-\mathrm{N}^{+}(\mathrm{R})_{3} \mathrm{Cl}$, where $\mathrm{R}$ is an alkyl group. Polymers having these strong acidic or basic groups easily decrease electrical resistance by sorbing water molecules. At the earliest stage of development of humidity sensors, polymer electrolytes themselves (homopolymers) were used as the sensing materials. For example, we have prepared various alkali salts of poly(2-acrylamido-2methylpropane sulfonic acid) PAMPS. These polymers were coated on an alumina substrate having a pair of gold electrodes. The impedance of these devices is plotted as a function of relative humidity in Fig. 1. The value of the impedance is in the order of $\mathrm{H}<\mathrm{Cs}<\mathrm{Rb}<\mathrm{K}<\mathrm{Na}<\mathrm{Li}$ salt. ${ }^{3)}$ On the other hand, the number of sorbed water molecules per sulfonate group is the largest for the acid form. The number of sorbed water molecules is in the order of $\mathrm{H}>\mathrm{Li}>$ $\mathrm{Na}>\mathrm{K}>\mathrm{Rb}>\mathrm{Cs}$ form. ${ }^{3)}$ When two different devices coated with the acid form and the Cs salt of PAMPS are stored in an atmosphere of $98 \% \mathrm{RH}$ at $25{ }^{\circ} \mathrm{C}$ for $500 \mathrm{hrs}$., the acid form deteriorates slightly while the Cs form does not as shown in Fig. 2. Noguchi et al. ${ }^{4)}$ also synthesized aliphatic ionene polymers, $-\mathrm{N}^{+}$ $\left(\mathrm{CH}_{3}\right)_{2}-\left(\mathrm{CH}_{2}\right)_{x}-\mathrm{N}^{+}\left(\mathrm{CH}_{3}\right)_{2}-\left(\mathrm{CH}_{2}\right)_{y^{-}}$which have quaternary nitrogens in their main chains. However, there is a problem that polymer electrolytes easily dissolve in water. As a result, humidity sensors using polymer electrolytes as humidity-sensitive materials cannot be operated at high humidity where dewdrops may 


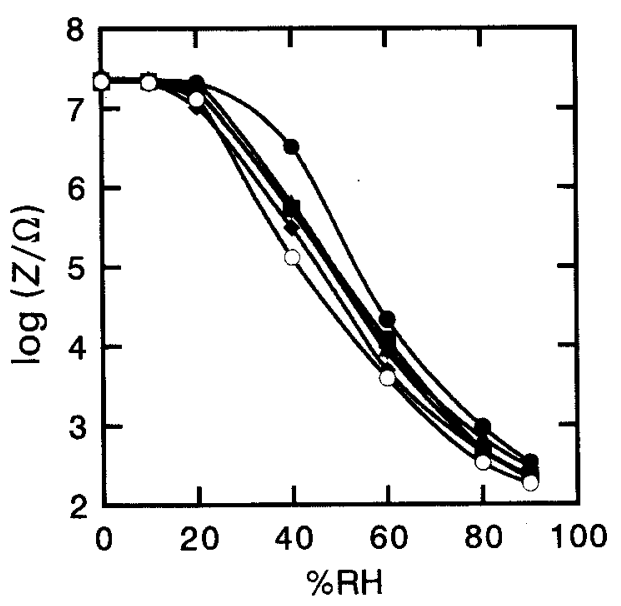

Fig. 1 Impedance of various alkali salts and the acid form of PAMPS as a function of relative humidity.

$\mathrm{Li},(\boldsymbol{\Delta}) \mathrm{Na},(\boldsymbol{\square}) \mathrm{K},(\boldsymbol{\nabla}) \mathrm{Rb},(\boldsymbol{)}) \mathrm{Cs}$ salt and (O) acid form of PAMPS. The immpedance was measured at $30{ }^{\circ} \mathrm{C}$ and $1 \mathrm{kHz}$.
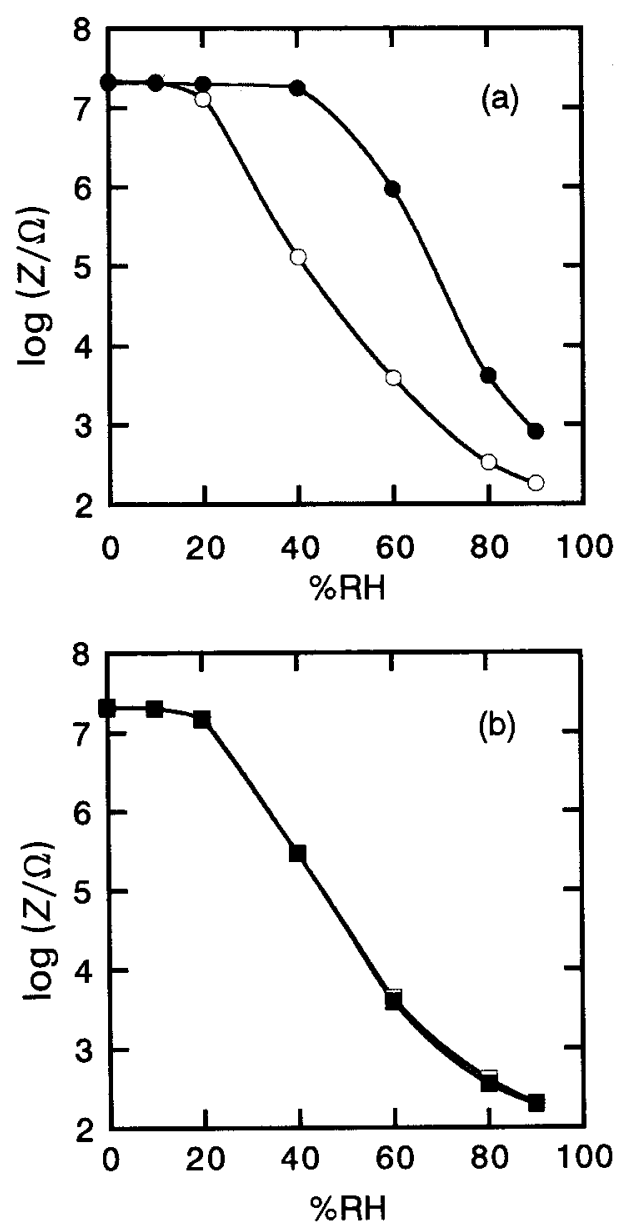

Fig. 2 The effect of humid atmosphere on the humidity dependence of impedance characteristics for the acid form and the Cs salt of PAMPS measured at $30^{\circ} \mathrm{C}$ and $1 \mathrm{kHz}$. (a) $(\bigcirc)$ virgin device using acid form of PAMPS, after 500 hrs storage in $98 \% \mathrm{RH}$ at $25{ }^{\circ} \mathrm{C}$.

(b) ( $\square$ ) virgin device using Cs salt of PAMPS, ( $\square$ ) after 500 hrs storage in $98 \% \mathrm{RH}$ at $25{ }^{\circ} \mathrm{C}$. appear on the sensor. Several methods can be used to overcome this problem. The methods of modifying the electrolytes to be insoluble in water are classified into three groups: 1) random copolymerization of a hydrophilic monomer with a hydrophobic monomer, 2) graft polymerization of a hydrophilic monomer onto a hydrophobic trunk polymer, 3) cross-linking of a hydrophilic polymer with an adequate cross-linking reagent. These methods of chemical modification of polymer electrolytes and their characteristics will be described in the following sections.

\section{3 Humidity sensors using random copolymers}

In order to make the polymer electrolyte durable in a highly humid atmosphere, various copolymers comprising a hydrophilic and a hydrophobic component have been synthesized and used as sensing materials. Takaoka et al. ${ }^{5}$ proposed a copolymer of 2-hydroxy3-methacryloyloxypropyl trimethylammonium chloride, which is hydrophilic, and a hydrophobic vinyl monomer such as styrene. A thin film of this copolymer was cast on an alumina substrate having a pair of gold electrodes. Kinjo and coworkers ${ }^{6,7)}$ prepared similar humidity sensors using a variety of ionic and nonionic monomers.

We have proposed another simpler method for the preparation of a copolymer. ${ }^{8)}$ Polystyrene was partly sulfonated to form a copolymer of hydrophobic styrene and hydrophilic styrene sulfonic acid. Poly-4vinylpyridine was also partly quaternized resulting in the formation of a kind of copolymer of hydrophobic vinylpyridine and hydrophilic quaternized vinylpyridine. The sensitivity to humidity depends on the degree of sulfonation or quaternization. Although fabrication of this type of sensor is simple, there is a disadvantage in that it is not completely resistant to water.

\section{4 Humidity sensor using graft copolymer}

When a hydrophilic monomer is graft-polymerized on a hydrophobic polymer film, a humidity-sensitive but water-insoluble copolymer film can be produced. As the trunk polymer, we used a polytetrafluoroethylene film or a microporous polyethylene film. Both films are completely insoluble in water even after being grafted with hydrophilic polymers. Styrene was graft-polymerized on the surface of a thick film of polytetrafluoroethylene (PTFE) (1 mm thick) by $\gamma^{-}$ ray irradiation. The polystyrene branch was then sulfonated to form styrene sulfonic acid.9) Some of the film was transformed into alkali salts. A humidity sensor of the surface type was prepared by depositing a pair of gold electrodes on the surface of the grafted film. The gap between the electrodes was $40 \mu \mathrm{m}$. The humidity dependence of impedance was measured for various alkali salts of sulfonate. 4-Vinylpyridine was also grafted on the same thick PTFE film followed by quaternization with alkyl halide. ${ }^{8)}$ A surface-type sensor was prepared. On the other hand, when a thin PTFE film (0.05 mm thick) was used as the trunk polymer, the grafted layer was formed through the bulk of the PTFE film. In this case, a sandwich type 
sensor was also fabricated by depositing gold electrodes on both surfaces of the grafted film. ${ }^{10)}$ These materials are useful for elucidating the sensing mechanism by varying the counter cation in the case of sulfonate and the counter anion in the case of pyridinium. At low humidities, the value of impedance for the sulfonate is in the order of $\mathrm{Li}$ salt $>\mathrm{Na}$ salt $=$ $\mathrm{K}$ salt $>$ acid form. However, at high humidities, the values of impedance for the three kinds of salts have a tendency to coincide. In the case of pyridinium, the iodide has the highest impedance, then the bromide, and the chloride has the lowest value. The difference in impedance among the three halides becomes smaller as the humidity increases. These results must be due to the increase in the number of charge carrier protons from the sorbed water as the humidity increases.

Polyethylene film was also used as the trunk polymer. In this case, however, porous films must be used instead of ordinary plain films. The grafted film made of polyethylene plain film showed significant hysteresis due to the slow sorption and desorption of water vapor. Consequently, a microporous polyethylene film was used. The thickness and porosity of the film were $100 \mu \mathrm{m}$ and $70 \%$, respectively. 2-Acrylamido-2methylpropane sulfonic acid (AMPS) ${ }^{11)}$ or 2-hydroxy3-methacryloyloxypropyl trimethylammonium chloride (HMPTAC) ${ }^{12,13)}$ was grafted on the microporous polyethylene by conventional catalytic initiation or ultraviolet irradiation. The graft polymerization takes place on the pore walls. Impedance was compared as a function of humidity for three grafted films with the same grafting ratio but synthesized by different initiation methods. It was found that the film grafted by ultraviolet pre-irradiation has the most homogeneous distribution of the grafted layer inside the film. In the case of AMPS, some of the grafted film was transformed into alkali salts. This procedure made the impedance of the film higher than the original acid form. The impedance is low in the order $\mathrm{H}<$ $\mathrm{K}<\mathrm{Na}<\mathrm{Li}$. This order is the same as that for PTFEgraft-polystyrenesulfonate. The response time is the shortest for the acid type (less than 2 minutes), followed by the $\mathrm{K}$ salt.

\section{5 Humidity sensors using hydrophobic polymers} chemically modified to have small ionic groups

Instead of grafting hydrophilic polymer branches onto a hydrophobic polymer, we have proposed the addition of small ionic groups on a hydrophobic polymer. For example, we have sulfonated microporous polyethylene with fuming sulfuric acid or chlorosulfuric acid. ${ }^{14)}$ We deposited gold electrodes on both sides of the film to prepare a humidity sensor. The sensor has good sensitivity to humidity and has a shorter response time than the grafted films described above. The only disadvantage of this method is that the strong sulfonating reagents slightly reduce the mechanical strength of the film. Xin et al. sulfonated polysulfone with chlorosulfonic acid to prepare a fast response, chemically stable, water-resistive and low cost sensor. ${ }^{15)}$ Commercially available ion exchange membranes have similar chemical structures but most of them are inadequate for humidity sensors because of the high resistivity at low humidities and significant hysteresis due to the slow response.

\section{6 Humidity sensors using cross-linked hydro-} philic polymers

When a polymer electrolyte is cross-linked with an appropriate cross-linking reagent (cross-linker), the molecular weight becomes extremely large, resulting in the modification of a water-soluble polymer into an insoluble one. There are two methods for preparing the cross-linked polymer.

The first method is polymerization of a mixture of a vinylic monomer and a cross-linker monomer having two vinyl groups. Hijikigawa et al..$^{16)}$ developed a humidity-sensitive film composed of polystyrene sul. fonate cross-linked with $\mathrm{N}$, N'-methylene bisacrylamide $\mathrm{CH}_{2}\left(\mathrm{NHCOCH}=\mathrm{CH}_{2}\right)_{2}$ by UV irradiation.

The second method is cross-linking of a polymer having reactive sites using a cross-linking reagent. We simultaneously cross-linked and quaternized poly4-vinylpyridine using $\alpha$, $\omega$-dichloroalkane as the cross-linker ${ }^{17)}$ as shown in Fig. 3. The mixture of poly-4-vinylpyridine and the cross-linker in methanol was cast on the substrate having interdigitated array electrodes, followed by heating. The humidity dependence of impedance and the degree of hysteresis depend on the alkyl group size of the cross-linker. It was found that 1,4-dichlorobutane is the best of the four cross-linkers having different alkyl groups. We also simultaneously cross-linked and quaternized poly(chloromethyl styrene) by the reaction with $\mathrm{N}, \mathrm{N}$, N',N'-tetramethyl-1,6-diaminohexane on a similar substrate. ${ }^{18)}$ The reaction scheme is illustrated in Fig. 4. The sensors proved to be durable in water.

Poly(2-hydroxy-3-methacryloxypropyl-trimethyl ammonium chloride)(HMPTAC) has a hydoxy group as well as a quaternary ammonium group. The hydroxy group can react with diisocyanate to form cross-linking bonds as illustrated in Fig. 5. The impedance versus relative humidity characteristics of this cross-linked film is almost the same as that of the non-cross-linked film. ${ }^{19)}$ In order to prepare a humidity sensitive film but completely resistive to water, the IPN (Interpenetrating Polymer Network) technique was applied to the fabrication of ionically conductive humidity sensors. An IPN film composed of crosslinked HMPTAC polymer and cross-linked ethyleneg-

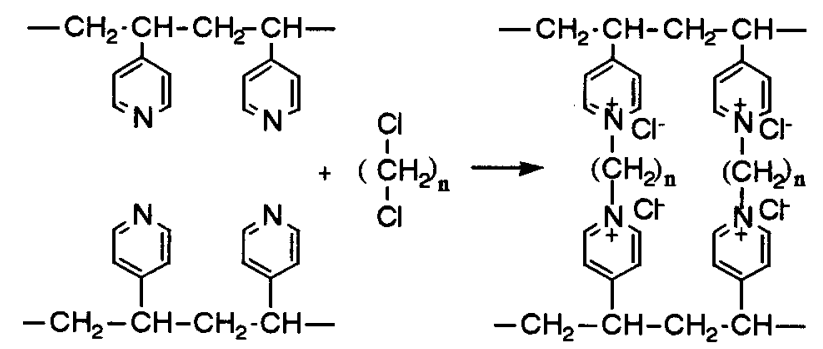

Fig. 3 Simultaneous cross-linking and quaternization of poly(4-vinylpyridine) with dichloroalkane. 
1ycol dimethacrylate(EGDMA) polymer as illustrated in Fig. 6 was formed on a substrate with intergiditated array electrodes. ${ }^{19)}$ The impedance of this sensor decreased from $10^{7}$ to $10^{2} \Omega$ with increasing humidity from 0 to $90 \% \mathrm{RH}$. In spite of cross-linking and interpenetrating with the hydrophobic component, the sensitivity to humidity was almost the same as that of an HMPTAC homopolymer sensor and the hysteresis was small. The characteristics of the IPN sensor changed little even after soaking in water for 2 hours. This technique of IPN can be applicable to the other ionically conductive polymer sensors when they are used at high humidity or when dew formation may occur. For example, 2-acrylamido-2-methylpropanesulfonic acid (AMPS) and polypropyleneglycol monomethacrylate (PGM) are copolymerized. Through the formation of bonding between $\mathrm{OH}$ groups of this copolymer with the $\mathrm{OH}$ groups of methylolmelamine, cross-linking bridges are then formed between the chains of the copolymer. At the same time, interpenetrating polymer networks are formed with poly(ethyleneglycol dimethacrylate) (EGDMA)

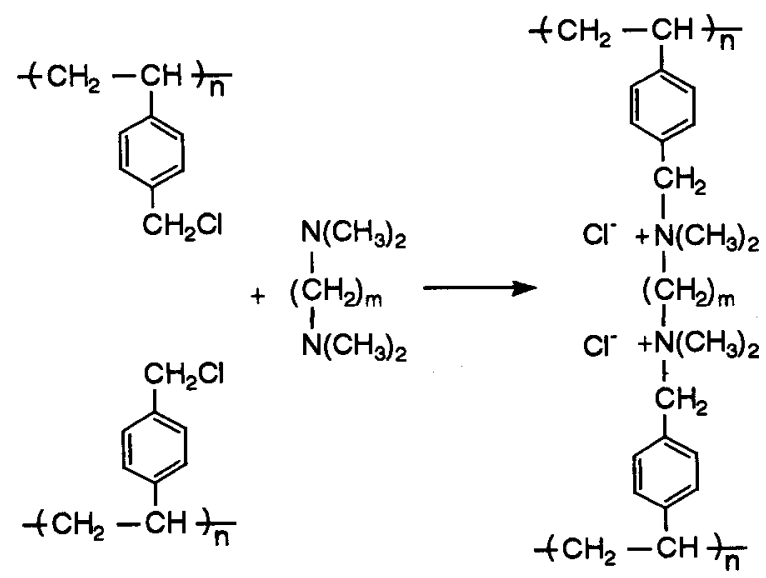

Fig. 4 Simultaneous cross-linking and quaternization of poly(chloromethyl styrene) with diaminoalkane.
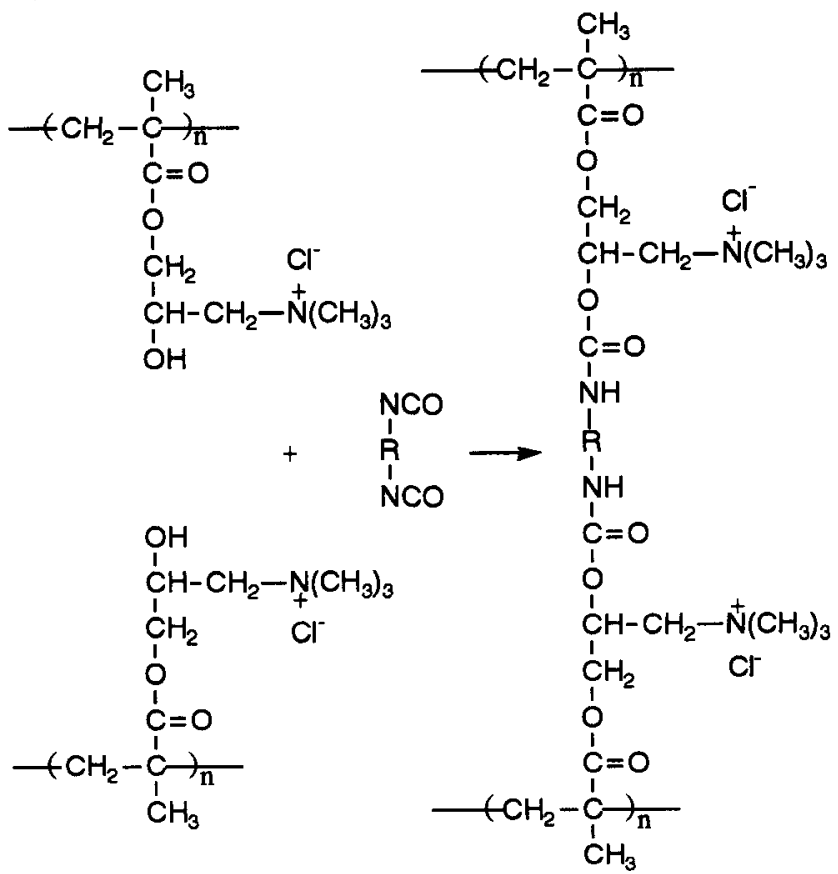

Fig. 5 Cross-linking of HMPTAC with diisocyanate.

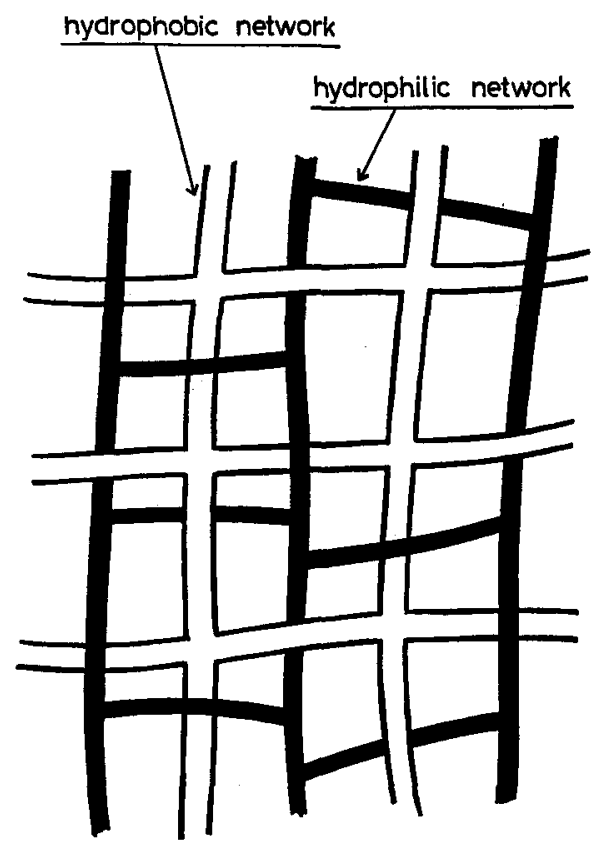

Fig. 6 Illustration of interpenetration polymer networks (IPN).

by mixing EGDMA monomer in the above casting solution. Because the homopolymer of AMPS is strongly hydrophilic and easily dissolves in water, it is inadequate for use in humidity sensors. However, through the formation of IPN with the hydrophobic cross-linking poly (ethyleneglycol dimethacrylate), a water-insoluble and humidity-sensitive-polymeric film is also formed. The response and recovery times of the sensor fabricated with IPN film are shorter than those of the sensor composed of the AMPS homopolymer.

On the other hand, we formed a cross-linked organopolysiloxane having hydrophilic groups such as $\mathrm{NH}_{2}, \mathrm{~N}^{+}\left(\mathrm{CH}_{3}\right)_{3} \mathrm{Cl}^{-}, \mathrm{SO}_{3} \mathrm{H}, \mathrm{OH}$ by the sol-gel method on a pressed silica gel or a sintered alumina plate. ${ }^{20)}$ The sensitivity to humidity is in the order $\mathrm{SO}_{3} \mathrm{H}>\mathrm{N}^{+}$ $\left(\mathrm{CH}_{3}\right)_{3} \mathrm{Cl}^{-}>\mathrm{NH}_{2}>\mathrm{OH}$. The sensors are washable in water. Another means of producing cross-linking between polymer chains is $\gamma$-ray irradiation. Rauen et al. ${ }^{21)}$ fabricated a humidity sensor by spin-coating an ionically conductive polymer, poly(dimethyldiallyl ammonium chloride), on a ceramic substrate, followed by cross-linking with gamma-irradiation using cobalt 60 .

\section{Capacitive-Type Humidity Sensors}

A cross-sectional view of a capacitive-type humidity sensor is schematically shown in Fig. 7. A parallel capacitor is formed on a substrate with a dielectric layer of sensing polymers. The sensor capacitance varies with the water adsorption and desorption by the sensing polymer based on the change in the effective dielectric constant. In order to fabricate capacitive-type humidity sensors, less hydrophilic polymers have been used. Cellulose derivatives were used for this type of sensor in the early steps of the development. For example, Sashida et al. prepared a 
sensor using cellulose acetate butyrate. ${ }^{22,23)}$ However, this material has several problems to be solved, such as hysteresis, drift over long-time use, sorption of some kinds of organic vapors. We have compared several types of cellulose derivatives and measured the change in dielectric constant caused by the sorbed water vapor. We found that the hysteresis is caused by the clustering of sorbed water molecules. ${ }^{24,25)}$ In order to minimize the clustering of sorbed water, we must choose a polymer in which the sorption sites of water molecules are scattered as much as possible and each site accommodates as few water molecules as possible.

\section{1 Humidity sensors using PMMA cross-linked with divinyl monomers}

We have chosen poly (methyl methacrylate) (PMMA) as one of the candidates for the capacitivetype humidity sensor because PMMA sorbs less water than cellulose acetate butyrate. Figure 8 shows the typical sensor characteristic of PMMA and cellulose acetate butyrate $(\mathrm{CAB})$ measured at $30{ }^{\circ} \mathrm{C}$ and 100 $\mathrm{kHz}$. The ratio of the capacitance at $\mathrm{X} \% \mathrm{RH}$ to the capacitance at $0 \% \mathrm{RH}$ is plotted on the ordinate. The electrical capacitance of the PMMA sensor changes almost linearly over the humidity range of $0-90 \% \mathrm{RH}$. As we expected, the hysteresis is less than $\pm 1 \%$. In addition, when we cross-linked MMA using a divinyl monomer, the hysteresis and the temperature coefficient were reduced. The durability on exposure to

\section{conductive paste}

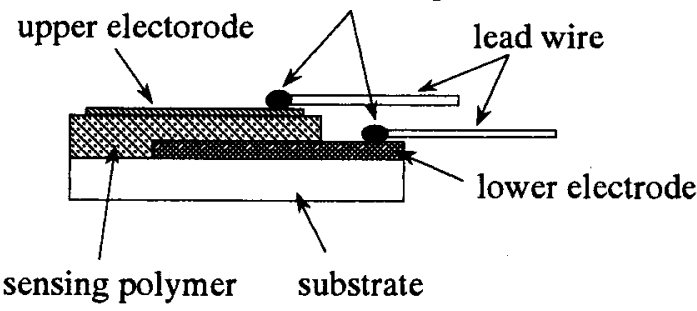

Fig. 7 Cross-sectional view of a capacitive-type humidity sensor.

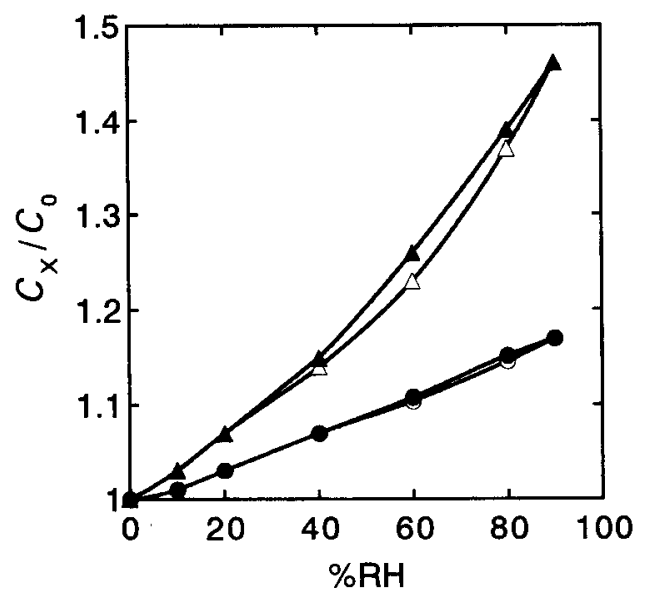

Fig. 8 Humidity dependence of the capacitance for the PMMA and $\mathrm{CAB}$ sensors measured at $30{ }^{\circ} \mathrm{C}$ and $100 \mathrm{kHz}$; $(\bigcirc)$ PMMA and $(\triangle) \mathrm{CAB}$. Closed symbols, humidification; open symbols, desiccation. organic vapors was also improved. ${ }^{26)}$

\section{2 Humidity sensors using polymers cross-linked by themselves}

It was proved that cross-linking of PMMA with some divinyl monomers was useful for preparing practical humidity sensors. However, mixing MMA with a cross-linking agent can induce phase separation and lead to an incomplete cross-linking reaction. Therefore, three kinds of unsaturated vinyl carboxylate, i.e., vinyl cinnamate, vinyl methacrylate, and vinyl crotonate, were chosen for novel sensing materials. These monomers have a carbonyl group as the polar site as does PMMA and are less hydrophilic. Furthermore, these monomers have two double bonds and have the possibility of cross-linking by themselves. ${ }^{27)}$ It was shown that the simple cross-linking process reflected not only the improvement of longterm stability but also the reproducibility of the sensor.

Various types of polyimides have been used to prepare the capacitive-type humidity sensors because of excellent thermal and chemical stability, low hygroscopicity and low solubility in common organic solvents. Most of the polyimides were those synthesized by polycondensation of a polyimide precursor. Ralston et al. investigated 13 polyimides of this type to determine the long-term stability at $85{ }^{\circ} \mathrm{C} / 85 \%$ $\mathrm{RH} .{ }^{28)}$ These polyimides may contain some unreacted sites and microvoids where volatile by-products such as water are released. In order to avoid these disadvantages, new compounds, that is, two kinds of acetylene-terminated polyisoimide oligomers, have been developed. ${ }^{29,30)}$ These oligomers have acetylenic bonds on both ends of the molecule and cyclotrimerize by heat treatment forming a cross-linked structure without evolving volatile by-products. The crosslinking produces a rigid film which does not contain microvoids and is very stable. The prepared sensor showed excellent sensor performance. The electrical capacitance of both sensors changed linearly over $10-90 \% \mathrm{RH}$ and their hysteresis was less than $1 \%$ $\mathrm{RH}$. Results of the resistance of the sensors to chemicals are shown in Table 1 . The magnitude of the sensor capacitance drift was converted into the apparent changes in relative humidity, $D$, by the following equation

$$
D(\% \mathrm{RH})=(C(\text { drift })-C(\text { init })) / S
$$

where $C$ (drift) is the capacitance after the sensor was exposed to various atmospheres and $C$ (init) is the initial capacitance before the test. Sensitivity, $S$,

Table 1 Summary of the resistance tests to chemicals.

\begin{tabular}{|c|c|c|}
\hline Test conditions & $\begin{array}{c}D / \% \mathrm{RH} \\
\mathrm{C}-\mathrm{PI} \text { sensor }\end{array}$ & $\begin{array}{c}D / \% \text { RH } \\
\text { C-FPI sensor }\end{array}$ \\
\hline Saturated acetone vapor: $20 \mathrm{~min}$ & 2.0 & 10.3 \\
\hline Formaldehyde & 2.0 & 2.5 \\
\hline Ethylene oxide gas & 4.0 & - \\
\hline Chlorine-type sterilizer & 3.0 & 2.5 \\
\hline Ampholytic surfactant & 2.5 & 4.5 \\
\hline
\end{tabular}


shown in this equation was obtained from the following equation,

$$
S\left(\mathrm{pF} \cdot \% \mathrm{RH}^{-1}\right)=\left(C_{90}-C_{10}\right) /(90-10),
$$

where $C_{90}$ and $C_{10}$ are the capacitance obtained at 90 $\% \mathrm{RH}$ and $10 \% \mathrm{RH}$, respectively. The crosslinked polyimide-based sensor (C-PI) proved to be resistant to chemicals. The sensor output was not affected even after exposing the sensor elements to saturated acetone vapor for $20 \mathrm{~min}$. The other fluorinated crosslinked polyimide-based (C-FPI) sensor demonstrated excellent long-term stability in harsh environments. Included in Table 2 for comparison are the results of long-term stability tests obtained for the C-PI sensor and the cross-linked PMMA (C-PMMA) sensor. The magnitude of the drift in the long-term stability tests was also calculated from Eq.(1). The drift of the C-FPI sensor was as small as $+1.1 \mathrm{pF}(D=3.8 \% \mathrm{RH})$ after $1000 \mathrm{~h}$ of exposure to $40{ }^{\circ} \mathrm{C}$ and $90 \% \mathrm{RH}$ atmospheres. A stable and reliable sensor usable in an office building and in a factory is achieved by using these sensors properly. The competitive adsorption behavior of water and $\mathrm{CO}_{2}$ gases on the C-PI film was also studied. ${ }^{31)}$ The result showed that the effect of coexistent $\mathrm{CO}_{2}$ on the sensitivity of C-PI sensor was quite small when we use it in an ordinary environment.

Another interesting material is poly (vinyl cinnamate) (PVCA). The cross-linked materials mentioned above were prepared by the radical reactions by heat treatment. However, there is an upper limit on the degree of cross-linking. PVCA undergoes an intermolecular photodimerization of the cinnamate moiety resulting in a highly cross-linked structure. The effect of the irradiation conditions on the degree of cross-linking and also on the sensing characteristics was examined. ${ }^{32)}$ The sensitivity was enhanced up to $c a .95 \%$ of the degree of cross-linking while the sensor showed a small hysteresis. Similar behavior was observed for the adsorption of organic vapors. When the degree of cross-linking attained more than $95 \%$, the film selectively adsorbed only molecules smaller than methanol, and the adsorption of the larger size organic vapors was greatly reduced as shown in Fig. 9. This result suggests that the higher cross-linking structure of the polymer reduces the cross-sensitivity to polar organic vapors. In fact, the effect of coexistent acetone vapor on the humidity sensing characteristic was considerably smaller for the sensor using the highly photocross-linked PVCA film. In addition, there is an optimum film thickness for preparing a selective humidity sensor. ${ }^{33)}$ This

Table 2 Summary of the long-term stability tests.

\begin{tabular}{lccc}
\hline Test conditions & $\begin{array}{c}D / \% \mathrm{RH} \\
\mathrm{C}-P I \text { sensor }\end{array}$ & $\begin{array}{c}D / \% \mathrm{RH} \\
\mathrm{C}-\mathrm{FPI} \text { sensor }\end{array}$ & $\begin{array}{c}D / \% \mathrm{RH} \\
\mathrm{C}-\mathrm{PMMA} \text { sensor }\end{array}$ \\
\hline $40{ }^{\circ} \mathrm{C}, 90 \% \mathrm{RH}: 1000 \mathrm{~h}$ & 20.6 & 3.8 & 10.5 \\
High temperature: $1000 \mathrm{~h}$ & $0.8\left(85{ }^{\circ} \mathrm{C}\right)$ & $-0.8\left(100{ }^{\circ} \mathrm{C}\right)$ & $-1.2\left(60{ }^{\circ} \mathrm{C}\right)$ \\
Room conditions: $1000 \mathrm{~h}$ & - & 0.9 & -0.7 \\
Outdoor conditions: $1000 \mathrm{~h}$ & - & $2.4(1486 \mathrm{~h})$ & 1.9 \\
\hline
\end{tabular}

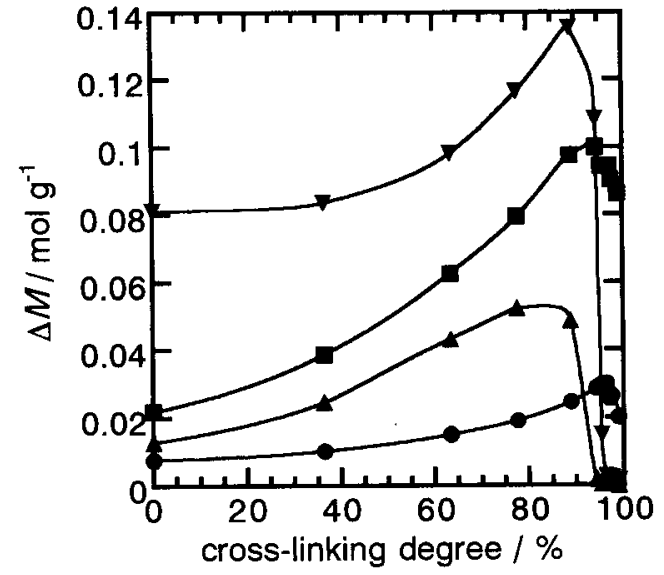

Fig. 9 Variation in the number of moles of adsorbed solute per gram of polymer at $8 \%$ rvp (relative vapor pressure) with the degree of crosslinking at $30{ }^{\circ} \mathrm{C}$; (O)

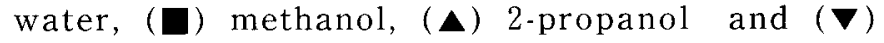
acetone.

sensor is useful for measuring humidity selectively in an atmosphere which contains organic vapors such as ethanol, acetone, etc. A novel double-layer structure sensor consisting of the photocross-linked PVCA and the PMMA film was proposed. The sensor reduced the disadvantage of both sensors by optimizing the thickness of the photocross-linked PVCA layer. ${ }^{34)}$

\section{Conclusions}

Among the various methods of preparing humidity sensitive polymeric materials for resistive-type humidity sensors, simultaneous cross-linking and introducing of hydrophilic groups onto the polymer on a substrate having interdigitated electrodes is one of the most promising methods. This sensor is fabricated only in a one-step procedure and the product has long-term stability in an atmosphere of high humidity. On the other hand, there are several useful materials for fabricating capacitive-type humidity sensors such as cross-linked poly(methyl methacrylate), polyimide formed from acetylene-terminated polyisoimide oligomer or cross-linked poly(vinyl cinnamate).

\section{Refereces}

1) Y. Sadaoka and Y. Sakai, Denki Kagaku (presently Electrochemistry), 52, 132 (1984).

2) Y. Sadaoka, Y. Sakai, and H. Akiyama, J. Mater. Sci., 21, 235 (1986).

3) N. Yonesato, M. Matsuguchi, and Y. Sakai, Proceedings of the 25th Chemical Sensor Symposium Japan, 85 (1997).

4) H. Noguchi and Y. Uchida, J. Mater. Sci. Lett., 8, 1278 (1989).

5) Y. Takaoka, Y. Maebashi, S. Kobayashi, and T. Usui, Jpn. Patent, 58-16467 (1983).

6) N. Kinjo, O. Ohara, T. Sugawara, and T. Tsuchitani, Polymer J., 15, 621 (1983).

7) S. Tsuchitani, T. Sugawara, N. Kinjo, and S. Ohara, Sensors and Actuators, 15, 375 (1988).

8) Y. Sakai, Y. Sadaoka, and H. Fukumoto, Sensors and 
Actuators, 13, 243 (1988)

9) Y. Sakai, Y. Sadaoka, and K. Ikeuchi, Sensors and Actuators, 9, 125 (1986).

10) Y. Sakai, Y. Sadaoka, M. Matsuguchi, Y. Kanakura, and M. Tamura, J. Electrochem. Soc., 138, 2474 (1991).

11) Y. Sakai, V. L. Rao, Y. Sadaoka, and M. Matsuguchi, Polymer Bull., 18, 501 (1987).

12) Y. Sakai, Y. Sadaoka, M. Matsuguchi, V. L. Rao, and M. Kamigaki, Polymer, 30, 1068 (1989).

13) Y. Sakai, Y. Sadaoka, M. Matsuguchi, and V. L. Rao, J. Mater. Sci., 24, 101 (1989).

14) Y. Sakai and Y. Sadaoka, Denki Kagaku (presently Electrochemistry), 53, 150 (1985).

15) Y. Xin and S. Wang, Sensors and Actuators A, 40, 147 (1994).

16) M. Hijikigawa, S. Miyoshi, T. Sugihara, and A. Jinda, Sensors and Actuators, 4, 307 (1983).

17) Y. Sakai, Y. Sadaoka, and M. Matsuguchi, J. Electrochem. Soc., 136, 171 (1989).

18) Y. Sakai, Y. Sadaoka, M. Matsuguchi, and H. Sakai, Sensors and Actuators B, 25, 689 (1995).

19) Y. Sakai, M. Matsuguchi, Y. Sadaoka, and K. Hirayama, J. Electrochem. Soc., 140, 432 (1993).

20) Y. Sakai, Y. Sadaoka, M. Matsuguchi, N. Moriga, and M. Shimada, Sensors and Actuators, 16, 359 (1989).

21) K. L. Rauen, D. A. Smith, W. R. Heineman, J. Johnson, R. Seguin, and P. Stoughton, Sensors and Actuators B, 17, 61 (1993).

22) T. Sashida, K. Akaba, and M. Kawarai, Kobunshi
Ronbunshu, 46, 499 (1989).

23) T. Sashida, K. Motosugi, K. Akaba, and M. Kawarai, Kobunshi Ronbunshu, 46, 795 (1989).

24) Y. Sadaoka, M. Matsuguchi, Y. Sakai, and K. Takahashi, J. Mater. Sci. Lett., 7, 121 (1988).

25) M. Matsuguchi, S. Umeda, Y. Sadaoka, and Y. Sakai, Sensors and Actuators B, 49, 179 (1998).

26) M. Matsuguchi, Y. Sadaoka, Y. Sakai, T. Kuroiwa, and A. Ito, J. Electrochem. Soc., 138, 1862 (1991).

27) M. Matsuguchi, Y. Sadaoka, Y. Nuwa, M. Shinmoto, and Y. Sakai, J. Electrochem. Soc., 141, 5614 (1994).

28) A. R. K. Ralston, C. F. Klein, P. E. Thoma, and D. D. Denton, Sensors and Actuators B, 34, 343 (1996).

29) M. Matsuguchi, Y. Sadaoka, K. Nosaka, M. Ishibashi, and Y. Sakai, J. Electrochem. Soc., 140, 825 (1993).

30) M. Matsuguchi, T. Kuroiwa, T. Miyagishi, S. Suzuki, T. Ogura, and Y. Sakai, Sensors and Actuators B, 53, 53 (1998).

31) M. Matsuguchi, H. Yokouchi, Y. Sadaoka, and Y. Sakai, Denki Kagaku (presently Electrochemistry), 63, 841 (1995).

32) M. Matsuguchi, M. Shinmoto, Y. Sadaoka, T. Kuroiwa, and Y. Sakai, Sensors and Actuators B, 34, 349 (1996).

33) M. Matsuguchi, M. Minomura, Y. Sadaoka, T. Kuroiwa, and Y. Sakai, Denki Kagaku (presently Electrochemistry), 64, 1107 (1996).

34) M. Matsuguchi, S. Kubo, and Y. Sakai, Electrochemistry, 67, 170 (1999). 